\title{
Los bonos-residencia en la Comunidad Valenciana: su efecto sobreel empleo
}

Rafael Granell Pérez y M aría Ángeles Tortosa Chuliáa

\section{Introducción}

Tradicionalmente, la financiación de las residencias de ancianos en España se ha venido realizando mediante dos mecanismos: la provisión directa de los centros públicos y los subsidios a los centros privados y del tercer sector. La Comunidad Valenciana de cidió incorporar en 1996 un nuevo instrumento de financiación, que hasta ahora no se había usado en España, denominado bonoresidencia. El principal propósito de este artículo es averiguar si la implantación de este instrumento ha colaborado a incrementar y mejorar las condiciones del empleo en este sector.

El esquema utilizado para efectuar este estudio ha sido el siguiente: en primer lugar, se presentan los problemas característicos del sector de las residencias de ancianos en España y en la Comunidad Valenciana. En segundo lugar, se revisa el origen y objetivos, así como el funcionamiento del sistema de bono-residencia en la Comunidad Valenciana. En tercer lugar se analizan los datos disponibles sobre el empleo y su relación con el bono, para finalizar con algunas conclusiones y recomendaciones futuras.

\section{Panorámica del sector de las residencias para mayores en la Comunidad Valenciana}

Tradicionalmente los servicios sociales para mayores en España se ofrecían a través de instituciones religiosas. A partir de los años 60, la participación del sector público fue aumentando y se impulsó la construcción y financiación de residencias públicas de gran tamaño, diseñadas para mayores en su mayoría válidos.

D espués de la transición democrática la demanda para obtener una plaza en estas residencias fue creciendo y pronto quedó de manifiesto que el número de estos centros era insuficiente. Ante esta situación la solución adoptada fue la de conceder subvenciones directas a los centros del tercer sector para que efectuaran ampliaciones. Sin embargo, la demanda seguía creciendo. Este hecho alentó a las empresas privadas a introducirse en el sector.

En los años 90, debido al compromiso político de mantener los gastos sociales y de continuar atendiendo las necesidades de los mayores (según el Plan Gerontológico), en algunas Comunidades Autónomas, como la Valenciana, las autoridades firmaron convenios con empresas privadas para que produjesen los servicios de las residencias públicas. N ace así un nuevo tipo de residencia pública denominada de «gestión integral».

A principios del siglo xxı en España, y en particular en la C omunidad Valenciana, nos encontramos con una oferta heterogé nea de residencias para mayores (públicas, privadas y del tercer sector) que todavía es insuficiente, dado el envejecimiento progresivo de la población. Una oferta que se caracteriza por la importancia de la iniciativa privada-tercer sector (cuentan con un 60\% de las plazas) (IM SERSO, CSISC, Univ. Autónoma, 2000).

El Plan Gerontológico Nacional de 1992 dejaba claro que uno de los objetivos prioritarios era incrementar el número de plazas en residencias de ancianos. El cuadro 1 muestra lo que se fue consiguiendo, según los datos disponibles de 1994 y 1999. A pesar de este aumento, la proporción de mayores que ocupaba 


\section{Cuadro 1}

Datos sobre la población mayor y sobre la oferta deresidencias en España y en la Comunidad Valenciana (1994-99)

\begin{tabular}{|c|c|c|c|}
\hline & \multicolumn{2}{|c|}{ España } & \multirow{2}{*}{$\begin{array}{c}\text { Comunidad Valenciana } \\
1999\end{array}$} \\
\hline & 1994 & 1999 & \\
\hline 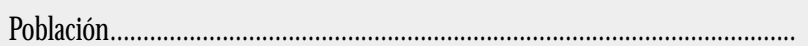 & 39.149 .465 & 39.806 .735 & 3.994 .446 \\
\hline 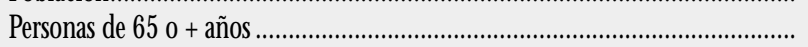 & 5.761 .715 & 6.858 .209 & 616.550 \\
\hline \% & $14,7 \%$ & $17,2 \%$ & $15,44 \%$ \\
\hline Residencias públicas. & - & 853 & 56 \\
\hline Residencias privadas y del tercer sector & - & 2.849 & 187 \\
\hline Total residencias & - & 3.702 & 243 \\
\hline \multirow[t]{2}{*}{ 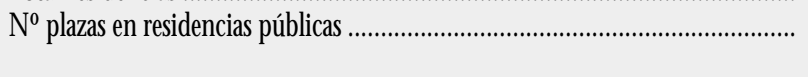 } & 53.161 & 68.455 & 4.317 \\
\hline & $(32,5 \%)$ & $(35,2 \%)$ & $(31,8 \%)$ \\
\hline \multirow{2}{*}{$\mathrm{N} \cong$ plazas en residencias privadas y del tercer sector } & 110.177 & 126.197 & 9.268 \\
\hline & $(67,5 \%)$ & $(64,8 \%)$ & $(68,2 \%)$ \\
\hline Total número de plazas & 163.338 & 194.652 & 13.585 \\
\hline 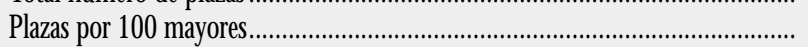 & $2,83 \%$ & $2,84 \%$ & $2,20 \%$ \\
\hline
\end{tabular}

Fuente: IM SERSO $(1995,1999,2000)$.

una plaza en las residencias permaneció estable durante este pe ríodo alrededor del 2,8\%. En la Comunidad Valenciana esta proporción se mantuvo en el 2,2\%. Como numerosas investigaciones comentan (Defensor Del Pueblo, 2000); (M inisterio de Trabaj0, 1999); (IM SERSO, CSIC y Universidad Autónoma, 2000, 2002), este resultado indica que, en el ámbito estatal y de las C omunidades Autónomas, se está lejos todavía en 1999 de lograr la proporción del 3,5\% del Plan Gerontológico para el año 2000, y que aún persiste una parte de demanda insatisfecha.

Como hemos explicado, esta diferencia entre oferta y demanda de plazas residenciales ha sido habitual en las últimas dé cadas, siendo conocidas las largas listas de espera para entrar en residencias públicas (unas 100.000 personas mayores esperando a mitad de los años 80) (In SERSO, 1987). Aunque se ha progresado en los últimos años, las listas de espera todavía persisten.

Estas listas de espera son inadmisibles y demasiado largas (ABELlán, 1997; 1999) si consideramos que no se trata de la primera opción de cuidado deseada por los mayores. Antes de entrar en una residencia prefieren la ayuda a domicilio, pero tropiezan con problemas de acceso o esta ayuda es insuficiente. Según los últimos datos del M inisterio (Im SERSO (b), 2000); (I M SERSO, CsIC, Universidad Autónoma, 2000; 2002) (M inisterio de Ciencia y Tecnología, CSIC, Fundación Pfizer, 2002), en la mayoría de las regiones la ayuda a domicilio sólo alcanza al 1,82\% de los mayores con una media de 4 horas/semana. La C omunidad Valenciana es la que en peor situación se encuentra ya que este porcentaje es sólo del 0,78\% de los ancianos. Ante este panorama de la ayuda a domicilio, los mayores buscan otras alternativas de cuidado y acaban solicitando la entrada en residencias.
En consecuencia, como esta demanda continúa creciendo, las autoridades de diferentes Comunidades Autónomas están buscando nuevas soluciones. Entre ellas se está potenciando que las residencias funcionen como centros de día o bien que permitan estancias temporales. Además, se continúan entregando subvenciones a los centros, y se añaden ayudas financieras directas a los usuarios y/o cuidadores (IM SERSO, 1999).

\section{El bono-residencia en la Comunidad Valenciana}

\subsection{Origen de los bonos}

Previa a la introducción de los bonos, la ayuda financiera pública al sector de las residencias en la Comunidad Valenciana consistía en: crear centros públicos; subvencionar a las instituciones del tercer sector; y firmar conciertos con centros privados para que produjesen el servicio.

Como la oferta pública no ha crecido tan rápido como se esperaba, estas medidas han propiciado que el número de plazas en residencias privadas y del tercer sector haya aumentado. A pe sar de esta nueva oferta, existen problemas de equidad en el acceso de los mayores a las residencias privadas. Q uienes esperan obtener una plaza pública no pueden sufragar mientras tanto 0 , 
como alternativa, el precio de una plaza privada. Por esta razón el sector público está buscando soluciones a este problema.

En la Comunidad Valenciana, la primera idea que se manejó fue la de construir más centros públicos, pero esta solución era muy costosa y útil solamente a largo plazo. A pesar de ello se dotó presupuesto para la construcción de nuevas residencias públicas (que suponen 9.000 nuevas plazas), de las que la mitad serán inauguradas próximamente (IM SERSO, C SIC, Univ. Autónoma, 2000).

0 tra solución era continuar concediendo subvenciones a los centros, pero esta vía tampoco suponía una rápida respuesta a la creciente demanda. Finalmente se pensó que, además de lo anterior, el sector público financiase plazas en centros privados. El método consistiría en subvencionar directamente a los usuarios mediante la entrega de bonos.

Los bonos se habían utilizado previamente en la ciudad de Valencia para financiar la educación infantil (G RANELL, 2002), y también se tenía conocimiento de alguna experiencia en el extranjero, como la ayuda a domicilio en la ciudad sueca de $N$ acka (N ACKA Ko m m un, 2000), y el sistema de bonos residencia implantado en Inglaterra durante el período de 1981-1993 (H aLL y EGGERS, 1995).

\subsection{0 bjetivos de los bonos}

Los bonos son un instrumento financiero que permite que se cumplan los objetivos y características de los cuasi-mercados (Le Grand y Bartlett 1993); (Calero, 1998); (Cullis y JoNES, 1997). Con los bonos-residencia los políticos valencianos pretenden cumplir los siguientes objetivos básicos:

a) Respetar las preferencias de los mayores y hacer más sencilla su elección de residencia.

b) $\mathrm{H}$ acer posible que aquellos mayores que no han podido entrar en una residencia pública debido a las largas listas de espera, tengan acceso a una plaza en una residencia privada.

c) $\mathrm{H}$ acer posible que aquellos mayores que previamente no podían pagar una plaza en una residencia privada obtengan la ayuda financiera complementaria para poder tener acceso a esas residencias.

Los objetivos hasta ahora mencionados tratan la libertad de elección y aligeran la espera y carga financiera. Aparte de esos objetivos, las normas de los bonos incluyen unas exigencias que afectan a los recursos materiales y personales de las residencias y que indican que los bonos pretenden que las residencias funcionen mejor. El que los centros deban cumplir unas estrictas condiciones sobre sus empleados sugiere que indirectamente los políticos pretenden utilizar el bono para promocionar el empleo en este sector.

\subsection{El funcionamiento de los bonos}

Los bonos fueron diseñados para ayudar financieramente a los mayores de 60 años que residen en la Comunidad Valenciana, necesitan entrar en una residencia y no tienen suficientes recursos económicos para poderlo efectuar.

Los bonos funcionan del siguiente modo. Los mayores reciben un bono mensual que entregan a la residencia que han escogido. Con la cantidad del bono, su aportación y la de sus hijos abonan el importe mensual del coste de la plaza en la residencia. D espués la residencia envía estos bonos a la Conselleria de Bienestar Social y los canjea por dinero.

La cantidad del bono es equivalente a la diferencia entre el coste de la plaza y lo que el usuario y sus hijos pueden pagar en función de su renta.

\section{BO NO (mensual) $=[($ Coste plaza, según tipo beneficiario) - (Aportación beneficiario + aportación familiar)]}

El coste de la plaza no es una cantidad homogénea. La fija el gobierno valenciano dependiendo de cuál sea su grado de necesidad (válido, asistido, semi-asistido o supra-asistido). Durante los años de experiencia de este programa esta clasificación ha ido variando y con ello también el coste de las plazas (ver cuadro 2).

En 1996, sólo existían tres tipos de plazas: válidos, semi-asistidos y asistidos, aunque los costes para la primera y segunda categoría eran los mismos. Pero, dado el aumento de solicitudes de personas con mayores dependencias y el agravamiento de las necesidades de quienes ya estaban admitidos, se tuvo que modificar esta clasificación ampliando la categoría de supra-asistido. El coste de las plazas es recal culado cada año incorporando el efecto de la inflación y de la introducción de nuevos equipos. Una condición importante de los bonos es que ninguna residencia puede solicitar más dinero a los beneficiarios del fijado por la normativa. Por tanto, este coste por plaza es un importe máximo.

Las aportaciones de los beneficiarios o de sus familiares son variables y directamente proporcionales a su nivel de renta. La aportación del beneficiario del bono es la misma que pagaría si hubiese obtenido una plaza en una residencia pública. Esta aportación está regulada por una norma regional, que estipula que la cantidad aportada por el anciano debe incrementarse conforme aumente su renta.

La aportación de los hijos fue introducida por la normativa de los bonos de 1997. La aportación de cada hijo es estimada a través de una tabla que adjunta la normativa, y en la que se considera el tamaño de su unidad familiar y su nivel de renta per capita mensual. La aportación de los hijos se produce cuando la renta per capita de su unidad familiar es superior al nivel del sa- 


\begin{tabular}{|c|c|c|c|c|c|}
\hline \multicolumn{6}{|c|}{ Cuadro 2} \\
\hline Situación de los mayores & 1996 & 1997 & 1998 & 1999 & 2000 \\
\hline Válido & 90.000 & 100.000 & 110.000 & 110.000 & 115.000 \\
\hline 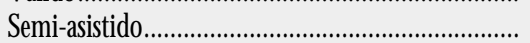 & 90.000 & - & 125.000 & 125.000 & 132.000 \\
\hline 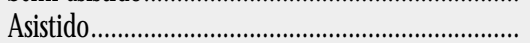 & 130.000 & 140.000 & 152.000 & 152.000 & 160.000 \\
\hline 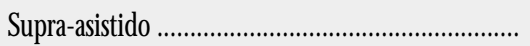 & - & 170.000 & 185.000 & 185.000 & 195.000 \\
\hline
\end{tabular}

Fuente: Conselleria de Bienestar Social.

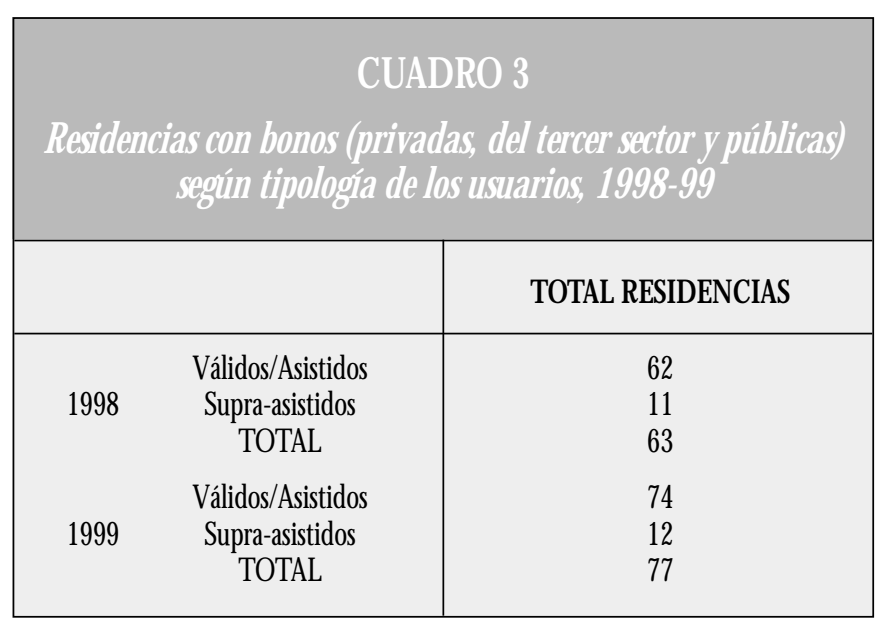

Fuente: Conselleria de Bienestar Social.

lario mínimo interprofesional (969.750 ptas. para 1999). Ésta es una característica del bono criticada porque podría estar favoreciendo a los ancianos que entran en residencias públicas, cuyos hijos no tienen que soportar ningún tipo de carga financiera.

El cuadro 3 muestra el número de residencias de la Comunidad Valenciana que reciben bonos. En total son 77 para 1999, lo que representa al rededor de un $31,6 \%$ del total de residencias de la Comunidad Valenciana. Aunque el sistema de bonos fue originariamente diseñado para aplicarse sólo a las residencias privadas y del tercer sector, el Gobierno Valenciano permitió la concesión de los bonos a una de las mayores residencias públicas de la $\mathrm{C}$ 0munidad como una experiencia piloto. A lo largo de estos años este experimento no se ha extendido a otras residencias públicas.

Las residencias que reciben bonos no están obligadas a ofrecer todas sus habitaciones a los beneficiarios de los bonos. Esto significa que pueden reservar algunas habitaciones a sus clientes privados. 0 tro aspecto importante es que el número de residencias que disponen de plazas para beneficiarios supra-asistidos no es elevado, a pesar del mayor énfasis por atender a este tipo de mayores según las últimas normativas. Sólo 12 de las residencias que participan en el programa del bono atendían a este tipo de ancianos en 1999. Un número, a nuestro juicio, insuficiente.

\section{El empleo en las residencias de ancianos}

\subsection{Argumentos a favor de la promoción del empleo}

Como hemos visto en el apartado anterior, la promoción de empleo es pretendida de forma indirecta por la normativa del bono. Esta preocupación por el empleo viene justificada por los argumentos que se exponen a continuación.

a) D ebe producirse un rápido aumento del número de residencias en un futuro próximo y es una buena oportunidad para regular el número y el tipo de empleados que se desea en ellas, así como las tareas que deben efectuar.

El ritmo de crecimiento del sector de las residencias viene marcado por la presión que la demanda ejerce sobre este sector. Una demanda que no está satisfecha con el tipo de cuidados que se venían dando en el antiguo modelo de macro-residencias públicas, y que pretende, no sólo mayor cantidad de residencias, sino también un servicio de calidad (entendido como aquel servicio que cuenta con los recursos materiales y personales adecuados).

Por otro lado, el sector sanitario también ejerce presión para que se den soluciones a las duraderas estancias de los ancianos en los hospitales de agudos. Situaciones, en su mayoría, provocadas por reingresos a consecuencia de la ausencia de cuidados sociales en torno al mayor. Ésta es una de las graves deficiencias denunciadas en la última evaluación del Plan Gerontológico: la falta de coordina- 


\begin{tabular}{|c|c|c|c|c|}
\hline \multicolumn{5}{|c|}{ 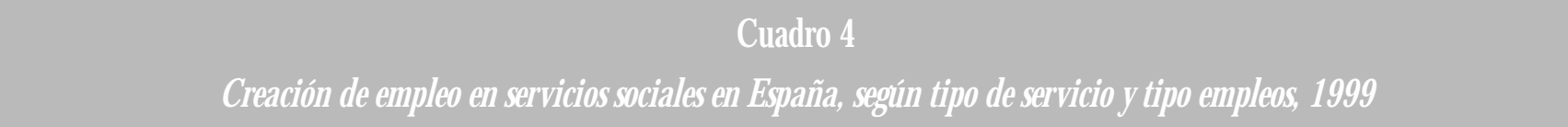 } \\
\hline & $\begin{array}{l}\text { Totales empleados } \\
\text { según tipo empleo }\end{array}$ & Tipos servicios & № empleados & $\begin{array}{l}\text { \% empleados por tipo } \\
\text { servicio/Total empleados }\end{array}$ \\
\hline Empleos a tiempo completo ..... & 21.577 & $\begin{array}{l}\text { Ayuda domicilio........................ } \\
\text { Atención diurna............................ } \\
\text { Residencias............................. } \\
\text { Alojamiento alternativo .............. }\end{array}$ & $\begin{array}{r}8.015 \\
628 \\
12.716 \\
218\end{array}$ & $\begin{array}{r}37,1 \\
2,9 \\
58,9 \\
1,1\end{array}$ \\
\hline Empleos a tiempo parcial .......... & 14.820 & $\begin{array}{l}\text { Ayuda domicilio ......................... } \\
\text { Atención diurna......................... } \\
\text { Residencias............................. } \\
\text { Alojamiento alternativo ................ }\end{array}$ & $\begin{array}{r}1.606 \\
458 \\
2.104 \\
93\end{array}$ & $\begin{array}{r}37,6 \\
10,7 \\
49,4 \\
2,2\end{array}$ \\
\hline Total empleados......................... & 25.838 & $\begin{array}{l}\text { Total empleados residencias/ } \\
\text { Total empleados.......................... }\end{array}$ & & \\
\hline
\end{tabular}

FUEN TE: I M SERSO (b), 2000; I M SERSO, CSIC, y Universidad Autónoma M adrid. 2000.

* Los datos son incompletos ya que sólo 10 de todas las Consejerías Sanidad y Servicios Sociales de las CCAA y Diputaciones Forales han aportado información sobre este tema. Por ejemplo, la Comunidad Valenciana no informa sobre este asunto.

ción del cuidado sanitario hospitalario de los ancianos con los servicios sociales (residencia y otros).

b) Los planes del gobierno central y autonómico sobre creación de empleo cuentan con un objetivo básico que es la erradicación del trabajo ilegal. En el sector de las residencias este es un problema importante. En la Comunidad Valenciana existían en 1999 unos 16.000 mayores atendidos en residencias ilegales (LLORENTE, 1999). Según nuestras estimaciones y atendiendo al número medio de empleados en residencias, esto supone que no están legalmente contratados y dados de alta en la seguridad social unos 6.800 trabajadores.

Además, estas residencias ilegales incorporan en la tarea de cuidar a los mayores a colectivos que no suelen estar dados de alta en la Seguridad Social, como al gunos inmigrantes u otros mayores con buena salud que se ofrecen como voluntarios. Unos colectivos que tampoco cuentan con la formación adecuada y/o no facilitan la comunicación y coordinación de las actividades que se desempe ñan en los centros.

c) Todavía queda mucho por hacer en cuanto a mejorar la formación de los empleados en servicios sociales destinados a los mayores (GONZÁLEZ, 2000). Sólo en estos últimos años se están incorporando nuevas especialidades para el cuidado de los mayores en los estudios universitarios y se ofrecen cursos de reciclaje sobre atención geriátrica desde las instituciones públicas.

Esta presión por mejorar los conocimientos de los empleados también es consecuencia del incremento del nú- mero de ancianos con necesidad de cuidados mayores y más especializados, como lo muestra el hecho de que el $32,21 \%$ de las personas mayores de 65 años presente discapacidades (M inisterio Trabajo, 2000).

d) 0 tros problemas pendientes de resolver sobre el personal en residencias y que son denunciados por ser la posible causa de problemas de maltrato al mayor son:

1) Desánimo ante la escasa dotación de personal en el centro, instalaciones y servicios adecuados.

2) Frustración/desmotivación de los trabajadores por el exceso de jornada laboral, guardias, turnos, baja remuneración salarial o imposibilidad de ascenso laboral.

3) Inexistencia de directrices u objetivos asistenciales definidos que llevan a un ritmo de trabajo anómalo 0 no sincronizado.

Unos resultados aproximados de cómo están influyendo las políticas hasta ahora adoptadas en el ámbito estatal en la creación de empleo en los servicios sociales en España se presentan en el cuadro $n-4$.

A través del mismo es posible observar que el mayor número de empleos creados se sitúa en la atención en residencias (un $57 \%$ del total empleos creados durante 1999 se centran en este sector). Según el tipo de empleos, tanto a tiempo completo como a tiempo parcial, el dominio del sector de las residencias frente al resto (58,9\% y 49,4\%, respectivamente) es evidente. Le sigue en importancia el número de empleos creados en la atención domiciliaria. 


\section{Cuadro 5}

\section{Condiciones de los trabajadores (1998-2001)}

1998 - 1999

N úmero mínimo de trabajadores por 100 usuarios.

Cualificación de los trabajadores.

Distribución del tiempo
35 para todo tipo centros

40 para centros con menos de 20 usuarios supra-asistidos,

45 para centros con 20-30 usuarios supra-asistidos,

50 para centros con más de 30 usuarios suprazasistidos.

En centros con supra-asistidos la plantilla debe contar con: médico, psicólogo, trabajador social, fisioterapeuta, ayudantes técnicos sanitarios y enfermeras.

El trabajo debe ser organizado de manera que se aseguren las 24 horas de cuidado en todo tipo de centros.

\section{Condiciones adicionales fijadas en el 2000-01}

Número mínimo y cualificación de los trabajadores
M ínimo de 35 trabajadores en todos los tipos de centros, de los que al menos $70 \%$ será personal cualificado de cuidado directo.

Centros con más de 100 usuarios deberán tener un médico y una enfermera trabajando $40 \mathrm{~h} / \mathrm{semana}$.

Centros con menos de 100 usuarios deberán tener un médico trabajando $1 \mathrm{~h} / \mathrm{sem} a n a$ por cada 10 usuarios y una enfermera trabajando $8 \mathrm{~h} / \mathrm{semana}$ por cada 10 usuarios.

40 para centros con menos de 20 usuarios supra-asistidos,

45 para centros con 20-45 usuarios supra-asistidos,

50 para centros con más de 45 usuarios suprazasistidos.

FUEN TE: Conselleria de Bienestar Social.

Por el examen de las cifras absolutas se desprende que el número de puestos de trabajo creado es escaso. A nuestro juicio, es debido básicamente a la limitada colaboración de al gunas C omunidades Autónomas a la hora de facilitar información sobre este tema.

0 tro dato a destacar negativamente es la alta proporción de empleos a tiempo parcial frente al total (43\%) que se han creado a lo largo de 1999. La explicación podría estar en que algunas re sidencias privadas-tercer sector cuentan con escasa dotación de personal porque su tamaño es reducido. Suelen atender a pocos ancianos (hasta un máximo de 50 son considerados centros de reducido tamaño por el IMSERSO) y son negocios familiares. Aproximadamente un 35\% de las residencias españolas para mayores tienen ese tamaño en 1998 [I M SERSO (b), 2000].

Esto explicaría que para este tipo de residencias no sea rentable contratar a personal especializado como médicos, podólogos, psicólogos, trabajadores sociales, etc., a tiempo completo. Las posibles necesidades en estos ámbitos de sus clientes las resuelven de varios modos: llamando a los servicios sanitarios públicos de la zona; solicitando puntualmente los servicios del profesional (y asumiendo los costes del servicio el propio anciano); o bien contratando directamente a estos especial istas a tiempo parcial.
Según la información del M inisterio de Trabajo (M inisterio Trabajo, 2000), ante presiones en la demanda, los empresarios del sector servicios (en el que se incluyen las residencias de mayores) y, sobre todo, aquellos centros cuyo tamaño es pequeño, recurren a la utilización de contratos temporales como la primera vía para salir del apuro. Además, en este estudio se constata que los principales motivos por los que no se contrata en el sector servicios son los altos costes salariales, la escasa ayuda oficial y la falta de formación adecuada de los trabajadores.

En resumen, como acabamos de ver, si los políticos valencianos pretenden mejorar el panorama del empleo en el sector de las residencias existe mucho por hacer. A continuación comprobaremos cómo influye la normativa del bono-residencia sobre el empleo.

\subsection{Efecto del bono-residencia sobre el empleo}

El sistema de bonos-residencia de la Comunidad Valenciana, como ya hemos comentado, ha asumido un nuevo e importante objetivo para el creciente sector de las residencias: el de promocionar empleo. Para ello, la normativa sobre los bonos incluye 


\begin{tabular}{|c|c|c|c|c|c|}
\hline \multicolumn{6}{|c|}{$\begin{array}{l}\text { Cuadro } 6 \\
\text { abajadores por } 100 \text { usvarios en centros con bonos (1999) }\end{array}$} \\
\hline & $\begin{array}{l}\text { Trabajadores de } \\
\text { cuidado directo }\end{array}$ & $\%$ & $\begin{array}{l}\text { Trabajadores que } \\
\text { no cuidan }\end{array}$ & $\%$ & $\begin{array}{l}\text { № TOTAL } \\
\text { trabajadores }\end{array}$ \\
\hline 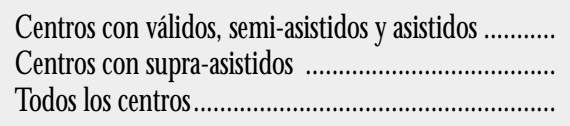 & $\begin{array}{l}38,63 \\
44,53 \\
40,22\end{array}$ & $\begin{array}{l}93,9 \\
95,8 \\
94,5\end{array}$ & $\begin{array}{l}2,51 \\
1,93 \\
2,35\end{array}$ & $\begin{array}{l}6,1 \\
4,2 \\
5,5\end{array}$ & $\begin{array}{l}41,14 \\
46,46 \\
42,57\end{array}$ \\
\hline
\end{tabular}

FUEN TE: Elaborado con datos de la Conselleria de Bienestar Social.

desde 1998 medidas para asegurar unas mejores condiciones de trabajo e intentar incrementar la calidad del servicio prestado en las residencias.

Las condiciones que se redactan en esta normativa afectan al número de empleados por usuario, a las cualificaciones que se requieren para el cuidado de cada tipo de usuario y a la distribución de horas de trabajo según el tipo de ancianos a cuidar (ver cuadro 5). Para verificar si estas condiciones habían sido cumplidas por las residencias perceptoras de bonos, solicitamos información a la Conselleria de Bienestar Social. El resultado aparece en los cuadros núms. 6 y 7.

En el cuadro 6 hemos analizado si el número medio de emplea dos disponibles en 1999 estaba por encima del nivel mínimo reque rido y también si contaban o no con la formación adecuada para el trabajo a desempeñar. En términos globales, este tipo de residencias tiene más de los 35 empleados por 100 usuarios (42 para el total de residencias). Un porcentaje medio menor de trabajadores en comparación con el que poseen las residencias públicas $(45,48)$.

Además, aquellas residencias con ancianos que presentan un alto nivel de dependencia tienen una ratio empleados/usuarios superior, entre los límites del 40 y 50\% que fija la normativa. También hemos comprobado que el porcentaje de personal directo es superior al 70\% (la proporción fijada por la normativa del bono del 2000).

D el cuadro 7 merece la pena destacar que las residencias con bonos tienen una proporción mayor de trabajadores sociales y sanitarios que las públicas. En cambio, en las públicas hay me nos especialistas sanitarios y más personal de administración y hostelería.

A parte, hemos observado que las residencias con bonos que cuentan con plazas de alta dependencia tienen el tipo de especialistas estipulado por la normativa (médicos, psicólogos, trabajadores sociales, fisioterapeutas y enfermeras). El resto de centros que asisten a ancianos válidos, asistidos o semi-asistidos cuentan con un número inferior de estos especialistas.

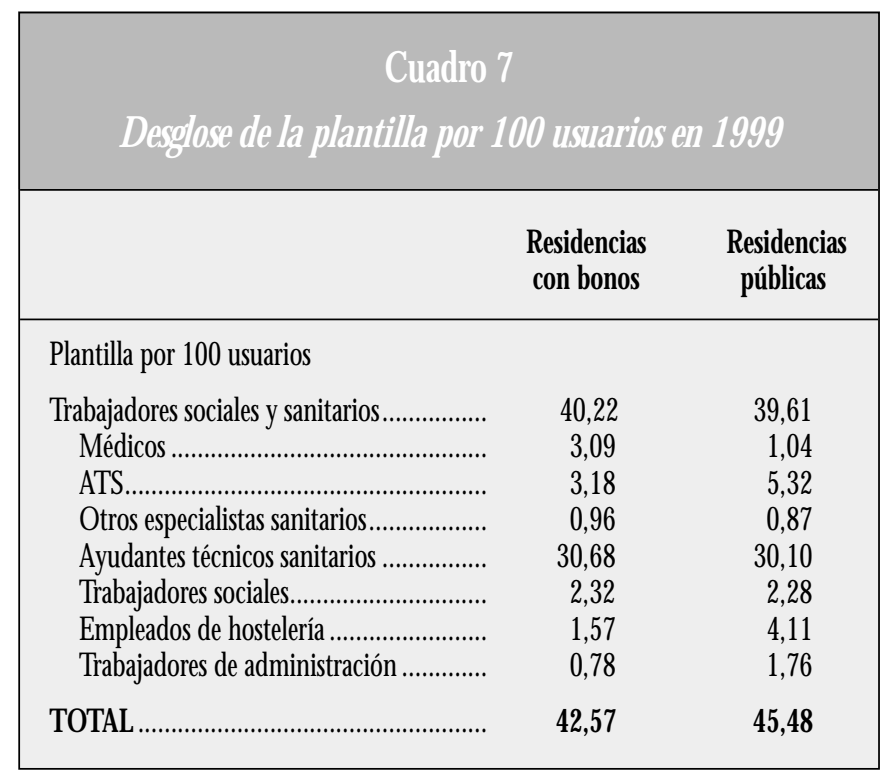

FUENTE: Elaborado con datos de la Conselleria de Bienestar Social.

O tra cuestión que nos interesa averiguar es si ha habido aumento del empleo en términos absolutos debido a los bonos. No se nos ha proporcionado un dato oficial sobre este aspecto, sólo contamos con la información proporcionada por la Asociación privada de residencias de mayores en la Comunidad Valenciana (AERTE), quien estima que el incremento en el empleo directo debido a las exigencias estipuladas por la normativa del bono es de 5,8 empleados por cada 100 usuarios.

Para estimar el efecto global sobre el empleo, hemos multiplicado este aumento por el número total de plazas disponibles en las residencias que cuentan con bonos (3.723 en 1999). Y esto significa que ha habido un incremento de aproximadamente 216 empleados en 1999.

Esta misma fuente (AERTE) también informa que los puestos de trabajo de especialistas son en gran parte a tiempo parcial, 
sobre todo en las residencias de pequeño tamaño. Los motivos de esta situación son:

- Las residencias centran sus esfuerzos en contratar trabajadores con formación en el área social y no sanitaria, ya que se consideran más como centros sociales que como sanitarios.

- No es rentable contratar a licenciados (sobre todo sanitarios) a tiempo completo porque sus salarios son elevados.

Por otro lado, las residencias dependen del buen funcionamiento de los bonos para crear empleo. Mientras el pago del bono por parte de la Administración se produzca a tiempo, sin retrasos, el centro puede hacer frente a sus gastos. En cambio, se ha comprobado que este funcionamiento es lento y los centros tienen problemas de impago con sus trabajadores y la Seguridad Social. Este hecho fue especialmente grave en el año 2000, y como resultado al gunas residencias decidieron abandonar el sistema del bono (A. G., 2000). Ello supuso, además, dejar sin plaza a los beneficiarios de los bonos de esas residencias. D esde entonces el sector empresarial está pidiendo un cambio en la gestión del bono y/o la concesión de otro tipo de ayudas.

\section{Conclusiones}

D esde hace unos años en la Comunidad Valenciana se ha implantado un nuevo sistema de financiación de las residencias de mayores. Se trata del bono-residencia. Cuando se introdujo este instrumento, los principales objetivos del programa consistían en reducir las largas listas de espera para entrar en una residencia y mejorar la igualdad de acceso a este tipo de servicios. Con el transcurso del tiempo al bono también se le está pidiendo que contribuya a crear y a mejorar el estado del empleo en el sector.

En este trabajo sólo hemos intentado constatar el cumplimiento del último de los objetivos que se le exige a los bonos-residencia. Y nuestras conclusiones son las siguientes:

- Una evaluación de la experiencia valenciana sobre los bonos muestra que se han creado nuevos empleos durante 1999 (el primer año después de la inclusión de este objetivo).

- No es posible averiguar con certeza con los datos disponibles si el empleo creado es suficiente o no para las necesidades del sector.

- Lo que está más claro es que el aumento del empleo es de bido también al interés de la iniciativa privada-tercer sec- tor por participar en este ámbito. Un aspecto que es favorablemente al entado por un sistema de bonos-residencia.

- Las residencias con bonos cumplen los requisitos de la normativa respecto al número y tipo de trabajadores. Situación que no hemos podido comprobar si cumplen las residencias privadas-tercer sector situadas fuera del programa.

- El tipo de empleo creado es, sobre todo, de atención directa.

- La presencia de trabajadores especializados y de mayor cualificación es mayor en las residencias con bonos que en las públicas.

- No pensamos que el bono haya supuesto cambios en cuanto al empleo ilegal, pues las residencias con bonos eran ya residencias legalizadas y que tenían empleados dados de alta, aunque sin el número o formación adecuados. Si estos centros aceptan los bonos saben que estarán bajo mayor control y tienen que estar dispuestos a ello. Los centros problemáticos son los que están al margen del bono, pues no se controlan tan exhaustivamente.

En este sentido, la Administración reconoce que el número de inspectores de Servicios Sociales es insuficiente $(6$ para toda la Comunidad Valenciana en 1999). Pero también se apunta que se van a adoptar medidas para solucionar este déficit. Estas me didas se resumen en regular un nuevo reglamento de inspección; en una ley de calidad de los servicios sociales; y en la contratación de empresas privadas, ajenas al sector, para efectuar el control de las residencias. Empresas que deberán ser finalmente auditadas por la Administración.

Es evidente que la Administración autonómica no está ejerciendo un fuerte control sobre las residencias ilegales. Si ejerciese ese control, ¿dónde colocarían a los 16.000 ancianos que se atienden en ellas? Recordemos que la Administración todavía es incapaz de cubrir la demanda con todos los instrumentos que ya están puestos en marcha.

¿Es el bono un buen instrumento para promocionar el empleo frente a otro tipo de regulación? Siempre y cuando una re gulación pública sobre este sector (subvenciones, bonos, norma para dar de alta a los centros, etc.) incorpore exigencias sobre el empleo para su puesta en marcha podrán ser útiles para promocionar el empleo. En el caso de la Comunidad Valenciana, sólo las normas del bono incorporan este tipo de condiciones, mientras que otras regulaciones, como las subvenciones a los centros, no las contienen. Por eso podemos decir que las residencias con bonos en la Comunidad Valenciana contribuyen a promocionar el empleo. 
Nota

* Profesores del D epartamento de Economía Aplicada, Universidad de Valencia.

Bibliografía

Abellán, A., 1997, «El alojamiento colectivo de personas mayores», Revista de $G e$ rontología, 7, 114-116.

AbelLán, A., 1999, «Problemas de vivienda y riesgo de institucionalización», Revista Multidisciplinar de Gerontología, 9 (4), 253-257.

AERTE. 2000, Guía de residencias privadas para mayores dela Comunidad Valenciana, AERTE, Valencia.

A.G. 2000, «Bienestar social lleva 4 meses sin pagar los bonos a las residencias de ancianos», D iario Levante, 04-05-2000.

Azn AR, M ., 1998, «Los servicios sociales públicos para mayores. Balance y prospectiva», D ocumentación Social, 112, 65-85.

Bartlett, W.; Propper, C.; W Illson, D. y Le Grand, J. 1994, Quasi-markets in the welfare state. The emerging findings, SAUS Publications, Bristol.

CALERO, J., 1998, Una evaluación de los cuasimercados como instrumento para la reforma del sector público, Fundación BBV, Bilbao.

CULLIS, J.G. y JONES, P.R., 1997, « os cuasimercados desde la perspectiva de la elección pública», H acienda Pública Española, 140 (1), 39-52.

D efensor del Pueblo 2000, La atención sociosanitaria en España: perspectiva gerontológica y otros aspectos conexos, $O$ ficina D efensor del Pueblo, Madrid.

Editorial, «M ás admite las carencias en la inspección y anuncia medidas de choque para controlar las residencias», D iario L evante, 18-11-1999.

González Góm EZ, S., 2000, «Formación en servicios sociales», Revista M ultidisciplinar de Gerontología, 10 (2), 106-111.

GranelL, R., 2002, «Education vouchers in Spain: The Valencian Experience», Education Economics, 2, 119-132.

H ALL, J. y EGGERS, W.D ., 1995, «H ealth and social services in the post-welfare state: are the vouchers the answer?», Reason Public Policy institute, Policy Study, 192.

In SERSO. 1987, Guía de centros de la tercera edad, InSERSO, M adrid.

I M SERSO . 1995, Guía directorio de centros para personas mayores, I m SERSO, M adrid.

IM SERSO(a). 2000, Las personas mayores y las residencias, I IM SERSO, M adrid.

I M SERSO (b). 2000, Servicios sociales disponibles en el Estado Español para las personas mayores: 1998, I M SERSO, M adrid.
I M SERSO, C SIC y Universidad Autónoma M adrid. 2000, Las personas mayores en España. Informe 2000, I M SERSO, M adrid.

I M SERSO, CSIC y Universidad Autónoma M adrid, 2002, Envejecer en España, I I SERso, Madrid.

JIMÉNEZ, E. y BARREIRO, F., 1999, «Los cheques-servicio como instrumentos de las políticas de empleo y bienestar», Ponencia de la Fundación CIREM , Fundación CIREM, Barcelona.

Kaufmann, A.E. y Frías, R. 1996, «Residencias: lo público y lo privado», Revista Española de Investigaciones Sociológic cas, 73, 81-104.

Le Grand, J. y BartLett, W., 1993, Q uasi-markets and social policy, The M acmillan Press, London.

LLORente, J.J., «Las personas mayores en el País Valencia. Aspectos de Sociología Crítica», 20/05/1999. D isponible en www.ctv.es/users/jjllorente

M IGUEL, J.A., 1998, «Balance de las políticas sociales con los mayores en España. Los planes gerontológicos. Análisis y perspectivas», D ocumentación Social, 112, 175-193.

M inisterio de Trabajo y Asuntos So Ciales. 1999, Informe de valoración del plan gerontológico estatal. 1992-1997, IM SERSO, M adrid.

M InISTERIO de Trabajo y Asuntos So ciales. 2000(a), Encuesta sobre discapacidades, deficienciasy estado de salud, 1999, IM SERSO, M adrid.

M inisterio de Trabajo y Asuntos Sociales. 2000(b), Encuesta de coyuntura laboral, Tablas de resultados del módulo anual de 1999, IM SERSO, M adrid.

Ministerio de Ciencia y Tecnología, Csic, Fundación Pfizer. 2002, Dependencia y necesidades asi stenciales de los mayores en España, una previsión a 2010, M inisterio de Ciencia y Tecnología, Madrid.

N Acka Kom m un. 2000, The homehelp services voucher, M unicipality of Nacka. Sweden.

PRATS, J., «El bono del consell para la tercera edad adolece de inseguridad jurídica», El País, 10-05-2000.

TORTOSA, M.A. and GranelL, R., 2002, «N ursing home vouchers in Spain: the Va lencian experiences, Ageing and Society, 22, 669-687. 
